\title{
Triglyceride glucose index influences platelet reactivity in acute ischemic stroke patients
}

\author{
Yinping Guo ${ }^{1}$, Jing Zhao ${ }^{1}$, Yi Zhang ${ }^{1}$, Lingshan Wu' ${ }^{1}$ Zhiyuan Yu ${ }^{1}$, Dan He ${ }^{2,3}$, Hao Huang ${ }^{1}$, Wensheng Qu ${ }^{1 *}$ and \\ Xiang LuO ${ }^{1 *}$
}

\begin{abstract}
Aim: Insulin resistance was reported to increase the risk of ischemic stroke, which can be assessed by the triglyceride glucose (TyG) index. However, it remains unclear whether the TyG index influences the platelet reactivity during the treatment of ischemic patients.

Methods: Ischemic stroke patients receiving dual antiplatelet therapy (DAPT) within $48 \mathrm{~h}$ onset were consecutively included. The TyG index was calculated as In (fasting triglyceride $[\mathrm{mg} / \mathrm{dL}] \times$ fasting glucose $[\mathrm{mg} / \mathrm{dL}] / 2$ ). The top quartile of TyG index was defined as insulin resistance. The platelet reactivity was assessed by thromboelastography. The platelet inhibition rate induced by arachidonic acid (AA) or adenosine diphosphate (ADP) was used to confirm the high residual on-treatment platelet reactivity (HRPR) to aspirin or clopidogrel, respectively. The association between TyG index and platelet reactivity was assessed by Kruskal-Wallis test. The independent risk factors of HRPR were determined by multivariate logistic regression analysis.
\end{abstract}

Results: A total of 1002 patients were included and divided into 4 groups by quartiles of the TyG index $(<2.02$; $2.02-2.27 ; 2.27-2.52 ; \geq 2.52)$. The findings demonstrated that the maximum intensity of the clot increased, but the AAinduced platelet inhibition rate decreased, depending on the TyG index quartiles. No significant difference was found in the ADP-induced platelet inhibition rate among groups. The prevalence of aspirin HRPR increased depending on the TyG index quartile. Unlike the non-insulin resistance group, the insulin resistance group was independently associated with aspirin HRPR (OR $=1.689,95 \% \mathrm{Cl} 1.14$ to $2.51, P=0.009)$.

Conclusions: In acute ischemic stroke patients taking DAPT, the elevation of the TyG index is associated with enhanced platelet reactivity and higher prevalence of aspirin HRPR. Insulin resistance assessed by the TyG index could be an independent risk factor for aspirin HRPR.

Keywords: Acute ischemic stroke, Dual antiplatelet therapy, Insulin resistance, TyG index

\section{Background}

Ischemic stroke is an important health problem worldwide and has the characteristics of high mortality and a high disability rate. Reducing the incidence of stroke and improving the prognosis of stroke are problems that

\footnotetext{
*Correspondence: qws0309@163.com; flydottjh@163.com

${ }^{1}$ Department of Neurology, Tongji Hospital, Tongji Medical College, Huazhong University of Science and Technology, Wuhan, Hubei 430030, People's Republic of China

Full list of author information is available at the end of the article
}

urgently need to be solved. Aspirin and clopidogrel are widely used in the acute phase treatment and secondary prevention of ischemic stroke [1,2]. They not only significantly reduce the mortality and disability rate but also effectively prevent the recurrence of stroke [3]. However, some patients with ischemic stroke will experience new ischemic events despite having received antiplatelet therapy [4-6]. One possible reason is the high residual on-treatment platelet reactivity (HRPR), which means 
a reduced platelet inhibition rate and the absence of an antiplatelet effect [7].

Insulin resistance is considered to be a significant risk factor of metabolic disorders [8], diabetes mellitus [9, $10]$, and atherosclerotic disease [11, 12]. In addition, more and more evidence shows that insulin resistance is common in ischemic stroke patients [13, 14]. Insulin resistance is independently related to the adverse clinical consequences of ischemic stroke, which can exacerbate the neurologic worsening during hospitalization and trigger the recurrence of ischemic stroke $[15,16]$. At present, the correlation between insulin resistance and platelet reactivity in acute ischemic stroke patients treated with dual antiplatelet therapy (DAPT) is unknown.

The gold-standard method for diagnosing insulin resistance is the hyperinsulinemic-euglycemic clamp (HIEC), which is not commonly used due to its cost and complexity [17]. Although widely used in research, the homeostatic model for assessing insulin resistance (HOMA-IR) is rarely used in clinical practice because of the nonuniform standards. Relatively, the triglyceride glucose index (TyG index) is a simple, economical, and reliable evaluation index of insulin resistance $[18,19]$. It has been confirmed that the TyG index is significantly coincident with HIEC and HOMA-IR in both non-diabetic and diabetic patients [20,21]. Assessed by the TyG index, insulin resistance was reported to be associated with carotid atherosclerosis, [22] coronary artery calcification [23], and ischemic stroke [13]. However, the correlation between the TyG index and the platelet reactivity in acute ischemic stroke patients receiving DAPT is unclear.

In order to disclose the impact of insulin resistance on HRPR, the correlation between the TyG index and platelet reactivity was evaluated in acute ischemic stroke patients who were receiving DAPT. The results may provide evidence for more effective individualized treatments during antiplatelet therapy.

\section{Methods}

\section{Study population}

Ischemic stroke patients who were admitted into the neurological department of Tongji Hospital were included retrospectively, from September 2013 and May 2019. Patients were included if: (1) over 18 years old; (2) with a diagnosis of acute ischemic stroke according to clinical symptoms and imaging (magnetic resonance/computer tomography); (3) receiving DAPT (clopidogrel $75 \mathrm{mg} /$ day and aspirin $100 \mathrm{mg}$ /day without loading dose) within $48 \mathrm{~h}$ of symptom onset, according to guidelines when diagnosed as acute minor ischemic stroke (with a National Institutes of Health Stroke Scale (NIHSS) score $\leq 3$ ) [24], high-risk transient ischemic attack (TIA, with a ABCD2 score $\geq 4$ ), or symptomatic severe stenosis (70-99\%) of a major intracranial artery (middle cerebral, carotid, vertebral, or basilar arteries) [2, 25], without evidences of cardioembolism. The exclusion criteria were as follows: (1) Without platelet reactivity testing after DAPT; (2) without fasting plasma glucose (FPG) or fasting triglycerides before DAPT; (3) any medications taken within the past 3 months that may affect blood coagulation function, such as cilostazol, warfarin, dabigatran, heparin, or factor Xa inhibitors (such as rivaroxaban); and (4) a history of malignant tumors, digestive diseases, or severe liver/ kidney/ blood-related diseases.

This study has been approved by the Tongji Hospital Ethics Committee (No. TJ-IRB20210107) and conducted according to the Declaration of Helsinki. According to ethic guidelines, the requirement of informed consent was waived since this study had no potential to harm the rights or welfare of subjects.

\section{Clinical assessments}

Clinical data included age, sex, smoking (defined as $\geq 1$ cigarette per day for 1 year or more), alcohol intake (defined as weekly alcohol intake exceeding $200 \mathrm{~g}$ for 1 year or more), a history of ischemic stroke/TIA, hypertension, hyperlipidemia, coronary heart disease (referring to prior angina pectoris or myocardial infarction), and diabetes mellitus (referring to glycosylated hemoglobin A1c (HbA1c) $\geq 6.5 \%$, or two-hour plasma glucose $\geq 11.1 \mathrm{mmol} / \mathrm{L}$ in an oral glucose tolerance test, or selfreported history of diabetes mellitus) [2, 26, 27]. Laboratory data included serum levels of fasting triglycerides (TGs), total cholesterol, high-density lipoprotein cholesterol (HDL-C), low-density lipoprotein cholesterol (LDL$\mathrm{C})$, creatinine, glomerular filtration rate (eGFR), platelet indexes, fasting plasma glucose (FPG), and HbA1c. All of these data were tested in a standard manner in the laboratory of the hospital within $24 \mathrm{~h}$ enrollment and before the DAPT.

The TyG index is calculated by the formula TyG index $=\mathrm{LN}$ [fasting TG $(\mathrm{mg} / \mathrm{dL}) \times \mathrm{FPG}(\mathrm{mg} / \mathrm{dL}) / 2$ ] [20] The top quartile $(\mathrm{Q} 4)$ of the TyG index is defined as insulin resistance.

\section{Platelet reactivity assessment}

It is believed that the inhibition plateau of platelet aggregation appeared 7 days after a regular dose of either aspirin or clopidogrel. Therefore, peripheral venous whole blood was collected 7 days later after the initiation DAPT with vacutainer tubes containing $3.2 \%$ sodium citrate and sodium heparin (Becton-Dickinson, San Jose, CA). The platelet reactivity was evaluated using Thromboelastography (TEG) Analyzer 5000 (Haemonetics Corporation, USA) within $1 \mathrm{~h}$ after the sample collection. The platelet 
reactivity induced by arachidonic acid (AA) and adenosine diphosphate (ADP) activators was tested according to the manufacturer's instructions. The maximum amplitude (MA) is the maximum intensity of the clot, which represents the maximum platelet function that can be stimulated in the blood sample. The $\mathrm{MA}_{\mathrm{ADP}}$ represents the ADP-induced clot strength while the $\mathrm{MA}_{\mathrm{AA}}$ represents that was induced by AA. The $\mathrm{MA}_{\text {fibrin }}$ represents the activator-induced clot strength (measurement of fibrin contribution) while the $\mathrm{MA}_{\text {thrombin }}$ represents that was induced by thrombin. The platelet inhibition rate induced by AA or ADP was calculated using the following formula: inhibition rate $(\%)=\left[\left(\mathrm{MA}_{\text {thrombin }}-\mathrm{MA}_{\mathrm{ADP}}\right.\right.$ or $\left.\left.\mathrm{MA}_{\mathrm{AA}}\right) /\left(\mathrm{MA}_{\text {thrombin }}-\mathrm{MA}_{\mathrm{fibrin}}\right)\right] \times 100 \%$. Aspirin HRPR was defined as AA\% $<50$, and clopidogrel HRPR was defined as $\mathrm{ADP} \%<30$ or $\mathrm{MA}_{\mathrm{ADP}}>47 \mathrm{~mm}[28,29]$.

\section{Statistical analysis}

Statistical analysis was completed using IBM SPSS 22.0 software (IBM Corp., Armonk, NY, USA). $P<0.05$ was considered as statistically significant difference. Categorical variables are shown as frequencies (percentages) and continuous variables as medians [interquartile range] for data with a skewed distribution. Patients were grouped by the TyG index quartiles and the clinical data were compared among groups using the Chi-square test or Kruskal-Wallis test.

For further analysis, patients were divided into aspirin HRPR and non-HRPR groups. Subsequently, the Chi-square or Mann-Whitney $U$ test was used to compare the demographic and clinical characteristics between groups. Potential confounders were adjusted by multivariable logistic models. The results were shown as adjusted odds ratios (ORs) with 95\% confidence intervals (CIs).

\section{Results}

\section{Demographics and clinical characteristics}

In the present study, 1002 patients were analyzed (Fig. 1). Values of the TyG index were divided into quartiles: Q1: TyG $<2.02(n=250) ; \mathrm{Q} 2:$ TyG $=2.02-2.27(n=251)$; Q3: TyG $=2.27-2.52(n=251)$; and Q4: TyG $\geq 2.52(n=250)$.

The baseline demographics and clinical characteristics of patients are described in Table 1. Comparing among different quartiles, a higher TyG index was related to a higher percentage of males, a history of diabetes mellitus, non-smoking cohorts, as well as the elevation of triglycerides, total cholesterol, HDL-C, LDL-C, creatinine, platelet count, fasting glucose, and HbA1c $(P<0.05)$.

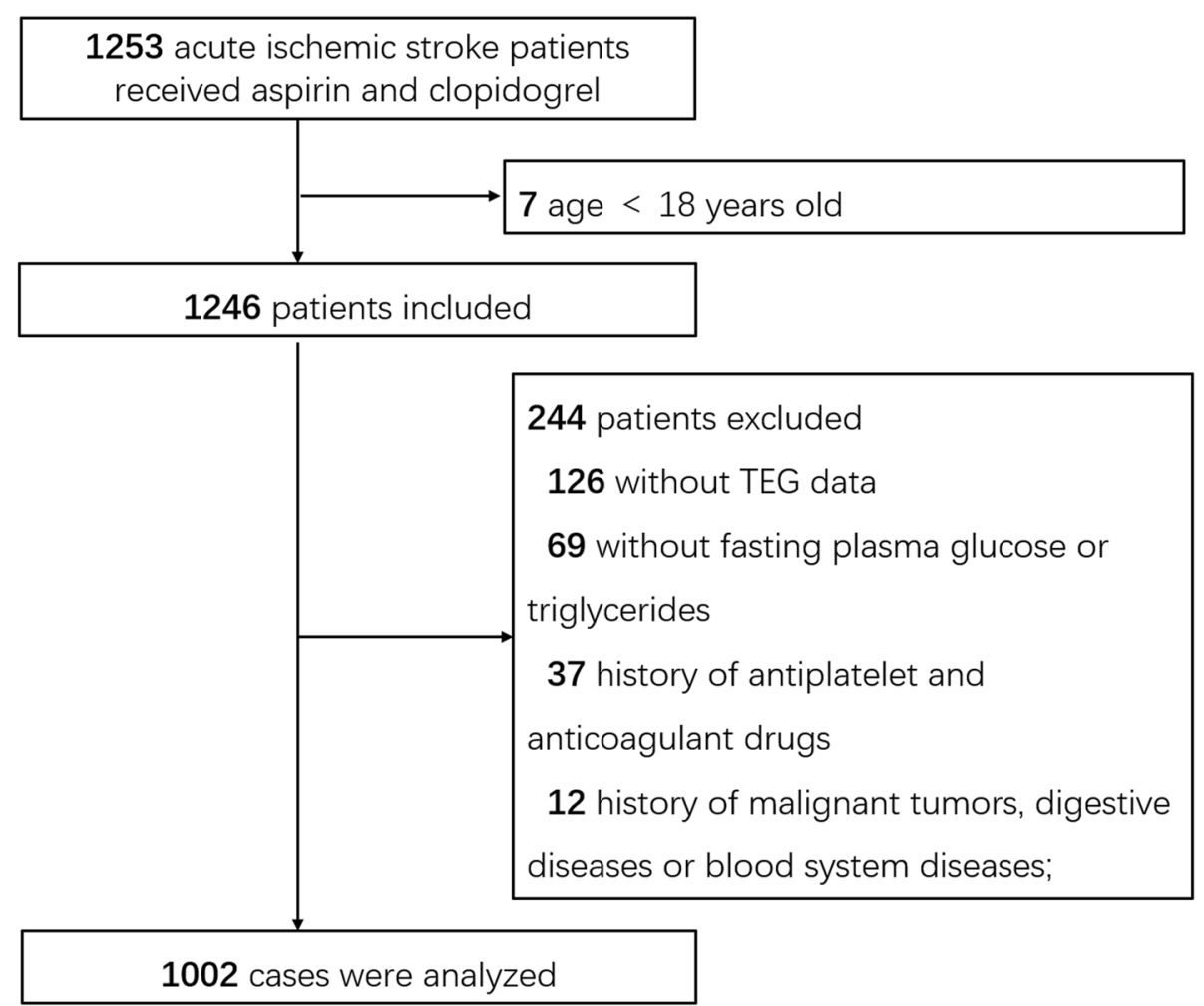

Fig. 1 Flowchart of patient selection. TEG: Thromboelastography 
Table 1 Patient baseline characteristics according to TyG index quartiles

\begin{tabular}{|c|c|c|c|c|c|}
\hline Characteristics & Q1 $(<2.02)(n=250)$ & Q2 (2.02-2.27) $(n=251)$ & Q3 $(2.27-2.52)(n=251)$ & $\mathrm{Q} 4(\geq 2.52)(n=250)$ & $P$ Value \\
\hline Age, y & $57.0[50.0-65.0]$ & $57.0[50.0-65.0]$ & $58.0[50.0-65.0]$ & $58.0[52.0-64.3]$ & 0.737 \\
\hline Male, $n(\%)$ & $207(82.8)$ & $183(72.9)$ & $170(67.7)$ & $166(66.4)$ & $<0.001$ \\
\hline Smoking, $n(\%)$ & $140(56.0)$ & $126(50.2)$ & $112(44.6)$ & $113(45.2)$ & 0.038 \\
\hline Alcohol intake, $n(\%)$ & $117(46.8)$ & $106(42.2)$ & $95(37.8)$ & $95(38.0)$ & 0.135 \\
\hline \multicolumn{6}{|l|}{ Medical history, $\boldsymbol{n}(\%)$} \\
\hline History of stroke/TIA & 49 (19.6) & $47(18.7)$ & $39(15.5)$ & $55(22.0)$ & 0.322 \\
\hline Hypertension & $173(69.2)$ & $158(62.9)$ & $177(70.5)$ & $170(68.0)$ & 0.288 \\
\hline Diabetes mellitus & $64(25.6)$ & 55 (21.9) & $70(27.9)$ & $149(59.6)$ & $<0.001$ \\
\hline Hyperlipidemia & $21(8.4)$ & $27(10.8)$ & $27(10.8)$ & $37(14.8)$ & 0.149 \\
\hline Coronary heart disease & $23(9.2)$ & $16(6.4)$ & $22(8.8)$ & $26(10.4)$ & 0.440 \\
\hline \multicolumn{6}{|l|}{ Biochemical indexes } \\
\hline Triglyceride (mmol/L) & $2.5[2.2-2.9]$ & $3.3[3.1-3.7]$ & $4.0[3.5-4.3]$ & $4.6[3.9-5.2]$ & $<0.001$ \\
\hline Total cholesterol (mmol/L) & $1.0[0.8-1.3]$ & $1.2[0.9-1.7]$ & $1.4[1.1-1.9]$ & $1.6[1.1-2.3]$ & $<0.001$ \\
\hline $\mathrm{HDL}-\mathrm{C}(\mathrm{mmol} / \mathrm{L})$ & $0.9[0.7-1.0]$ & $0.9[0.8-1.1]$ & $0.9[0.8-1.1]$ & $1.0[0.8-1.1]$ & $<0.001$ \\
\hline LDL-C (mmol/L) & $1.3[1.1-1.6]$ & $2.0[1.7-2.3]$ & $2.5[2.1-2.9]$ & $2.9[2.3-3.6]$ & $<0.001$ \\
\hline Creatinine $(\mu \mathrm{mol} / \mathrm{L})$ & $74.0[65.0-87.0]$ & $71.0[61.0-83.0]$ & $72.0[62.0-83.0]$ & $71.0[59.0-84.0]$ & 0.041 \\
\hline $\mathrm{eGFR}\left(\mathrm{mL} / \mathrm{min} / 1.73 \mathrm{~m}^{2}\right)$ & $94.8[80.6-105]$ & 96.1 [84.9-104] & $92.3[83.1-103]$ & 95.4 [81.4-103] & 0.580 \\
\hline Platelet count $\left(\times 10^{9} / \mathrm{L}\right)$ & 211 [169-247] & 209 [175-258] & $211[178-250]$ & 220 [184-259] & 0.039 \\
\hline PDW (fL) & 13.5 [11.9-15.1] & $13.1[11.7-14.9]$ & 13.3 [11.9-15.3] & $13.2[11.7-14.9]$ & 0.617 \\
\hline P-LCR (\%) & $33.8[28.2-40.4]$ & $32.4[27.0-39.7]$ & $32.0[26.6-39.7]$ & $32.9[27.0-38.5]$ & 0.210 \\
\hline Fasting glucose (mmol/L) & $4.9[4.6-5.3]$ & $5.1[4.8-5.5]$ & $5.4[5.1-6.1]$ & $7.1[5.8-10.0]$ & $<0.001$ \\
\hline HbA1c (\%) & $5.7[5.5-6.2]$ & $5.7[5.5-6.1]$ & $5.8[5.5-6.3]$ & $6.7[5.8-10.3]$ & $<0.001$ \\
\hline
\end{tabular}

Data given as $n$ (\%) or median [interquartile range]

Abbreviations: TyG index triglyceride glucose index, Q1-Q4 TyG index quartiles, TIA transient ischemic attack, HDL-C high-density lipoprotein cholesterol, LDL-C lowdensity lipoprotein cholesterol, eGFR glomerular filtration rate, PDW platelet distribution width, $P$ - LCR platelet large cell ratio, HbAc1 glycosylated hemoglobin A1C

\section{Relationship between TyG index and platelet reactivity}

Comparisons among different quartiles revealed that patients with a higher TyG index had higher levels of MA (the median values were 61.8, 62.1, 62.7, and $63.1 \mathrm{~mm}$ in each quartile respectively, $P=0.004)$ and lower levels of AA\% (the median values were 99.0, $97.4,96.2$, and $93.0 \%$ in each quartile respectively, $P<0.001)$. However, there was no significant difference of either $\mathrm{MA}_{\mathrm{ADP}}$ or $\mathrm{ADP} \%$ levels among the quartiles (Table 2).
There were $12.97 \%$ patients who were identified as having aspirin HRPR. The prevalence of aspirin HRPR was increased in the higher quartile of the TyG index (7.2\% vs. $12.0 \%$ vs. $14.7 \%$ vs. $18.0 \%, P=0.003)$. Relatively, there was no significant difference in the prevalence of the clopidogrel HRPR among the TyG index quartiles (Table 2).

\section{Risk factors of aspirin HRPR}

To disclose the risk factors for aspirin HRPR, the included patients were divided into two groups,

Table 2 Platelet reactivity and HRPR according to TyG index quartiles

\begin{tabular}{|c|c|c|c|c|c|}
\hline Characteristics & Q1 $(<2.02)(n=250)$ & Q2 (2.02-2.27) $(n=251)$ & Q3 (2.27-2.52) $(n=251)$ & $\mathrm{Q} 4(\geq 2.52)(n=250)$ & $P$ Value \\
\hline $\mathrm{MA}(\mathrm{mm})$ & $61.8[57.8-65.2]$ & $62.1[58.2-65.5]$ & $62.7[59.2-66.3]$ & $63.1[59.7-66.5]$ & 0.004 \\
\hline$M A_{A D P}(m m)$ & $30.9[18.1-42.5]$ & $31.0[16.8-41.3]$ & $31.6[18.6-42.5]$ & $30.0[18.3-42.2]$ & 0.645 \\
\hline ADP\% $(\%)$ & $58.4[39.1-81.4]$ & $60.2[40.1-85.1]$ & $58.2[35.5-82.3]$ & $61.6[41.9-86.0]$ & 0.306 \\
\hline $\mathrm{AA} \%(\%)$ & $99.0[87.2-100]$ & $97.4[83.7-100]$ & $96.2[77.9-100]$ & $93.0[70.0-98.9]$ & $<0.001$ \\
\hline Clopidogrel HRPR, n (\%) & $40(16.0)$ & $43(17.1)$ & $54(21.5)$ & $51(20.4)$ & 0.338 \\
\hline Aspirin HRPR, n (\%) & $18(7.2)$ & $30(12.0)$ & $37(14.7)$ & 45 (18.0) & 0.003 \\
\hline
\end{tabular}

Data given as $n$ (\%) or median [interquartile range]

Abbreviations: TyG index triglyceride glucose index, Q1-Q4 TyG index quartiles, $M A$ maximum amplitude, $M A_{A D P} A D P$-induced platelet-fibrin clot maximum amplitude, $A D P \%$ inhibition rate of adenosine diphosphate (ADP), $A A \%$ inhibition rate of arachidonic acid (AA), HRPR high residual on-treatment platelet reactivity 
non-HRPR and HRPR. The baseline demographic data, clinical characteristics, and laboratory data were compared. It demonstrated that the history of stroke/TIA, triglycerides, LDL-C, fasting glucose, and TyG index quartiles were significantly different between groups (Table 3). After adjustment for the potential confounders (history of stroke/TIA), compared with that in the Q1 quartile, the TyG index in either Q2, Q3 or Q4 was independently related to the aspirin HRPR in acute ischemic stroke patients taking DAPT (Q2: $\mathrm{OR}=1.76$, 95\% CI 0.95-3.26; Q3: OR=2.29, 95\% CI 1.27-4.16; $\mathrm{Q} 4: \mathrm{OR}=2.81,95 \%$ CI $1.57-5.01 ; P<0.05)$. Moreover, insulin resistance, presented by the Q4 quartile of the TyG index, was confirmed as an independent risk factor for the aspirin HRPR when comparing with the noninsulin resistance cohort $(\mathrm{Q} 1+\mathrm{Q} 2+\mathrm{Q} 3)(\mathrm{OR}=1.69$, 95\% CI 1.14-2.51, $P=0.009$ ).

\section{Discussion}

In the present study, it was found that as the TyG index increased, the prevalence of diabetes, blood lipids, and glucose levels were elevated significantly. The TyG index was significantly correlated with the increased platelet reactivity and decreased response to aspirin in acute ischemic stroke patients who received DAPT; the insulin resistance was an independent risk factor for aspirin HRPR. This suggested that insulin resistance may increase the progression and recurrence of ischemic stroke by decreasing the platelet reactivity to aspirin in patients receiving DAPT.

It is well known that insulin resistance is closely related to hyperglycemia and abnormal lipid metabolism. In the process of body glucose balance, insulin resistance affects the expression and activity of glucose transporters, thereby increasing the accumulation of glucose in

Table 3 Risk factors for aspirin HRPR

\begin{tabular}{|c|c|c|c|}
\hline Characteristics & Non-HRPR $(n=872)$ & $\operatorname{HRPR}(n=130)$ & $P$ Value \\
\hline Age (y) & $57.0[50.0-65.0]$ & $58.0[50.8-66.0]$ & 0.545 \\
\hline Male, $n(\%)$ & $632(72.5)$ & $94(72.3)$ & 0.968 \\
\hline Smoking, $n(\%)$ & $428(49.1)$ & $63(48.5)$ & 0.895 \\
\hline Alcohol intake, $n$ (\%) & $361(41.4)$ & $52(40.0)$ & 0.763 \\
\hline \multicolumn{4}{|l|}{ Medical history, $n$ (\%) } \\
\hline History of stroke/TIA & $155(17.8)$ & 35 (26.9) & 0.013 \\
\hline Hypertension & $591(67.8)$ & $87(66.9)$ & 0.846 \\
\hline Diabetes mellitus & $295(33.8)$ & $43(33.1)$ & 0.865 \\
\hline Hyperlipidemia & 99 (11.4) & $13(10.0)$ & 0.648 \\
\hline Coronary heart disease & $77(8.80)$ & $10(7.70)$ & 0.667 \\
\hline \multicolumn{4}{|l|}{ Laboratory data } \\
\hline Triglyceride (mmol/L) & $3.46[2.81-4.18]$ & $3.84[3.09-4.40]$ & 0.001 \\
\hline Total cholesterol (mmol/L) & $1.23[0.92-1.07]$ & $1.30[1.02-1.88]$ & 0.141 \\
\hline $\mathrm{HDL}-\mathrm{C}(\mathrm{mmol} / \mathrm{L})$ & $0.92[0.79-1.07]$ & $0.92[0.77-1.09]$ & 0.902 \\
\hline LDL-C (mmol/L) & 2.08 [1.49-2.68] & $2.33[1.80-3.00]$ & 0.001 \\
\hline Creatinine $(\mu \mathrm{mol} / \mathrm{L})$ & $72.0[62.0-85.0]$ & $73.0[62.8-83.0]$ & 0.763 \\
\hline $\mathrm{eGFR}\left(\mathrm{mL} / \mathrm{min} / 1.73 \mathrm{~m}^{2}\right)$ & $94.4[82.5-103]$ & $94.4[80.9-105]$ & 0.891 \\
\hline Platelet count $\left(\times 10^{9} / \mathrm{L}\right)$ & $211[177-251]$ & $221[180-264]$ & 0.060 \\
\hline PDW (fL) & $13.3[11.8-15.0]$ & $13.3[11.9-15.1]$ & 0.935 \\
\hline P-LCR (\%) & $33.0[27.3-39.7]$ & $32.1[27.0-38.3]$ & 0.593 \\
\hline Fasting glucose (mmol/L) & $5.29[4.84-6.29]$ & $5.55[5.05-6.75]$ & 0.010 \\
\hline $\mathrm{HbA1c}(\%)$ & $5.80[5.50-6.50]$ & $5.90[5.60-6.80]$ & 0.297 \\
\hline $\mathrm{MA}(\mathrm{mm})$ & $62.4[58.7-66.0]$ & $63.6[58.2-66.1]$ & 0.337 \\
\hline TyG index quartiles & & & $<0.001$ \\
\hline Q1 (<2.02) & $232(26.6)$ & $18(13.8)$ & \\
\hline Q2 (2.02-2.27) & $221(25.3)$ & $30(23.1)$ & \\
\hline Q3 (2.27-2.52) & $214(24.5)$ & $37(28.5)$ & \\
\hline $\mathrm{Q} 4(\geq 2.52)$ & $205(23.5)$ & $45(34.6)$ & \\
\hline
\end{tabular}

Data given as $n$ (\%) or median [interquartile range]

Abbreviations: TIA transient ischemic attack, HDL-C high-density lipoprotein cholesterol, $L D L-C$ low-density lipoprotein cholesterol, eGFR glomerular filtration rate, $P D W$ platelet distribution width, $P$ - $L C R$ platelet large cell ratio, $H b A c 1$ glycosylated hemoglobin $A 1 c, M A$ maximum amplitude, TyG index triglyceride glucose index, Q1-Q4 TyG index quartiles 
the circulatory system and forming hyperglycemia [30]. Similarly, during the process of lipid metabolism, insulin resistance increases the synthesis of triglycerides and their release, leading to increased triglycerides in plasma [31]. Both high blood glucose and abnormal lipid metabolism can affect insulin activity and exacerbate the insulin resistance. The TyG index has been proved to consistent with the "gold standard" for diagnosing insulin resistance, HIEC [17]. In the present study, it was demonstrated that a higher TyG index is related to diabetes mellitus, triglycerides, total cholesterol, and LDL-C, which suggests that the TyG index is a reliable alternative index for evaluating the insulin resistance.

Previous studies have found that insulin resistance is usually associated with platelet activation [32-34]. Insulin resistance could cause not only chronic inflammation but also endothelial dysfunction, which contributes to the increase of platelet adhesion and aggregation. In addition, studies have found that, by improving insulin resistance, the use of insulin sensitizers or strict blood sugar control could reduce the risk of thrombosis [35, 36]. In the present study, the highest levels of MA, representing increased platelet activation, were found in the highest quartile of the TyG index (insulin resistance group). However, further research is still needed to clarify the mechanism.

Previous studies have found that aspirin can decrease the risk of ischemic events by almost $10 \%$ or $20 \%$ in patients with or without diabetes, respectively [37]. Relative to non-diabetic patients, aspirin is less effective at the prevention of cerebrovascular diseases in diabetic patients [38]. The aspirin HRPR is thought to contribute to this variation. Thromboxane A2 is an important positive feedback mediator involved in platelet activation. However, aspirin could irreversibly inhibit the transformation of arachidonic acid to thromboxane A2, leading to the reduction of platelet aggregation [39]. Previous studies have shown that insulin resistance could activate other regulatory pathways of platelets, instead of thromboxane A2, to reverse the platelet inhibition of aspirin [40, 41]. Here, it was found that patients with a higher TyG index had higher levels of AA\%. Insulin resistance, presented by the highest quartile of the TyG index, was an independent risk factor for aspirin HRPR, which was supported by a previous study using a different method [42].

It is still controversial whether insulin resistance can influence the clopidogrel HRPR. In one previous study involving 237 patients with recent ischemic stroke or TIA receiving aspirin or/and clopidogrel, insulin resistance assessed by HOMA-IR was found to be related to HRPR, which was not specially restricted by clopidogrel resistance [43]. In another study including 66 ischemic stroke or TIA patients with clopidogrel treatment, insulin resistance assessed by HOMA-IR was associated with clopidogrel resistance tested by multiple electrode aggregometry [44]. The present study demonstrated that insulin resistance cannot influence the clopidogrel HRPR significantly. Relative to aspirin, clopidogrel resistance is more easily affected by confounding factors such as genes polymorphisms, which made it difficult to correlate insulin resistance with clopidogrel resistance. Further study of their relationship is needed.

Some limitations of this study should be addressed. First, since it was a retrospective study, some potential confounding factors for platelet reactivity, such as proton pump inhibitors, statins, or heterogeneity of metabolic genes, could not been evaluated. Second, measurement error of the system could not be completely ruled out since only one fasting test of triglyceride and glucose was performed. Third, the research subjects were from a cohort of Chinese patients, so extending these results to other cohorts may require careful interpretation and further researches.

\section{Conclusion}

In conclusion, we found that in acute ischemic stroke patients taking DAPT, the TyG index is associated with increased platelet activity and elevated prevalence of aspirin HRPR. The insulin resistance assessed by the TyG index could be an independent risk factor for aspirin HRPR. These results partly explained the higher risk of stroke recurrence in patients with insulin resistance despite receiving intensive antiplatelet therapy. Hence, personalized treatment strategies should be implemented by considering the TyG index in order to reduce the recurrence of ischemic events.

\section{Abbreviations \\ DAPT: Dual Antiplatelet Therapy; TyG: Triglyceride glucose; HRPR: High High residual on-treatment platelet reactivity; ADP: Adenosine Diphosphate; AA: Arachidonic Acid; TIA: Transient Ischemic Attack; HIEC: Hyperinsulinemic-eug- lycemic clamp; HOMAIR: Homeostatic model for assessing insulin resistance; FPG: Fasting plasma glucose; HDL-C: High-density lipoprotein cholesterol; LDL- C: Low-density lipoprotein cholesterol; eGFR: Glomerular filtration rate; PDW: Platelet distribution width; P-LCR: Platelet large cell ratio; HbAc1: Glycosylated hemoglobin A1c; MA: Maximum amplitude.}

\section{Acknowledgements}

We thank Dr. Guo Li and Mr. Renjie Feng for kindly participating in the data analysis.

\section{Authors' contributions}

YPG, JZ, YZ and HH collected the clinical data. YPG, DH and LSW processed statistical data. YPG, ZYY and WSQ drafted and revised the manuscript. XL and WSQ designed and guided the study. All authors read and approved the final manuscript. 


\section{Funding}

This study was supported by the National Nature Science Foundation of China (81771341 to X. Luo), Key Research and Development Program of Hubei Province (2020BCA070 to X. Luo), the Application Foundation Frontier Special Project of Wuhan Science and Technology Bureau (2020020601012226 to X. Luo). $X$. Luo designed and guided the study. And this study was also supported by the Natural Science Foundation of Guangdong Province (2018A030313820 to D. He). D. He processed statistical data.

\section{Availability of data and materials}

All data generated or analyzed during this study are included in this published article.

\section{Declarations}

\section{Ethics approval and consent to participate}

This study has been approved by the Tongji Hospital Ethics Committee (No. TJIRB20210107) and conducted according to the Declaration of Helsinki. According to ethic guidelines, the requirement of informed consent was waived since this study had no potential to harm the rights or welfare of subjects.

\section{Consent for publication}

Not Applicable.

\section{Competing interests}

The authors declare that there is no conflict of interest.

\section{Author details}

${ }^{1}$ Department of Neurology, Tongji Hospital, Tongji Medical College, Huazhong University of Science and Technology, Wuhan, Hubei 430030, People's Republic of China. ${ }^{2}$ Department of Neurology, The First Affiliated Hospital, Sun Yat-sen University, Guangzhou, Guangdong, China. ${ }^{3}$ Guangdong Provincial Key Laboratory for Diagnosis and Treatment of Major Neurological Diseases, National Key Clinical Department and Key Discipline of Neurology, Guangzhou, Guangdong, China.

Received: 27 May 2021 Accepted: 18 October 2021

Published online: 26 October 2021

\section{References}

1. Powers WJ, Rabinstein AA, Ackerson T, et al. Guidelines for the early Management of Patients with Acute Ischemic Stroke: a guideline for healthcare professionals from the American Heart Association/American Stroke Association. Stroke. 2018;49(3):e46-e110.

2. Kernan WN, Ovbiagele B, Black HR, et al. Guidelines for the prevention of stroke in patients with stroke and transient ischemic attack: a guideline for healthcare professionals from the American Heart Association/ American Stroke Association. Stroke. 2014;45(7):2160-236.

3. Chen ZM, Jiang LX, Chen YP, et al. Addition of clopidogrel to aspirin in 45,852 patients with acute myocardial infarction: randomised placebocontrolled trial. Lancet. 2005;366(9497):1607-21.

4. Arsava EM, Kim GM, Oliveira-Filho J, et al. Prediction of early recurrence after acute ischemic stroke. JAMA Neurol. 2016;73(4):396-401.

5. Amarenco P, Lavallee PC, Labreuche J, et al. One-year risk of stroke after transient ischemic attack or minor stroke. N Engl J Med. 2016;374(16):1533-42.

6. Lovett JK, Dennis MS, Sandercock PA, Bamford J, Warlow CP, Rothwell PM. Very early risk of stroke after a first transient ischemic attack. Stroke. 2003:34(8):e138-40.

7. Sofi F, Marcucci R, Gori AM, Giusti B, Abbate R, Gensini GF. Clopidogrel non-responsiveness and risk of cardiovascular morbidity. An updated meta-analysis. Thromb Haemost. 2010;103(4):841-8.

8. Khan SH, Sobia F, Niazi NK, Manzoor SM, Fazal N, Ahmad F. Metabolic clustering of risk factors: evaluation of triglyceride-glucose index (TyG index) for evaluation of insulin resistance. Diabetol Metab Syndr. 2018;10:74.

9. Zhang M, Wang B, Liu Y, et al. Cumulative increased risk of incident type 2 diabetes mellitus with increasing triglyceride glucose index in normal-weight people: The Rural Chinese Cohort Study. Cardiovasc Diabetol. 2017;16(1):30.

10. Lee DY, Lee ES, Kim JH, et al. Predictive value of triglyceride glucose index for the risk of incident diabetes: a 4-year retrospective longitudinal study. PLoS One. 2016;11(9):e0163465.

11. Lee EY, Yang HK, Lee J, et al. Triglyceride glucose index, a marker of insulin resistance, is associated with coronary artery stenosis in asymptomatic subjects with type 2 diabetes. Lipids Health Dis. 2016;15(1):155.

12. Lee $\mathrm{SB}, \mathrm{Ahn} \mathrm{CW}$, Lee BK, et al. Association between triglyceride glucose index and arterial stiffness in Korean adults. Cardiovasc Diabetol. 2018;17(1):41.

13. Shi $W$, Xing $L$, Jing $L$, et al. Value of triglyceride-glucose index for the estimation of ischemic stroke risk: insights from a general population. Nutr Metab Cardiovasc Dis. 2020;30(2):245-53.

14. Jing J, Pan $Y$, Zhao $X$, et al. Insulin resistance and prognosis of nondiabetic patients with ischemic stroke: the ACROSS-China study (abnormal glucose regulation in patients with acute stroke across China). Stroke. 2017:48(4):887-93.

15. Zhou Y, Pan Y, Yan $H$, et al. Triglyceride glucose index and prognosis of patients with ischemic stroke. Front Neurol. 2020;11:456.

16. Ago T, Matsuo R, Hata J, et al. Insulin resistance and clinical outcomes after acute ischemic stroke. Neurology. 2018;90(17):e1470-7.

17. Yusuf S, Hawken S, Ounpuu S, et al. Obesity and the risk of myocardial infarction in 27000 participants from 52 countries: a case-control study. Lancet. 2005;366(9497):1640-9.

18. Simental-Mendia LE, Rodriguez-Moran M, Guerrero-Romero F. The product of fasting glucose and triglycerides as surrogate for identifying insulin resistance in apparently healthy subjects. Metab Syndr Relat Disord. 2008;6(4):299-304.

19. Hong S, Han K, Park CY. The triglyceride glucose index is a simple and low-cost marker associated with atherosclerotic cardiovascular disease: a population-based study. BMC Med. 2020;18(1):361.

20. Guerrero-Romero F, Simental-Mendia LE, Gonzalez-Ortiz M, et al. The product of triglycerides and glucose, a simple measure of insulin sensitivity. Comparison with the euglycemic-hyperinsulinemic clamp. J Clin Endocrinol Metab. 2010;95(7):3347-51.

21. Vasques AC, Novaes FS, de Oliveira MS, et al. TyG index performs better than HOMA in a Brazilian population: a hyperglycemic clamp validated study. Diabetes Res Clin Pract. 2011;93(3):e98-e100.

22. Irace C, Carallo C, Scavelli FB, et al. Markers of insulin resistance and carotid atherosclerosis. A comparison of the homeostasis model assessment and triglyceride glucose index. Int J Clin Pract. 2013;67(7):665-72.

23. Kim MK, Ahn CW, Kang S, Nam JS, Kim KR, Park JS. Relationship between the triglyceride glucose index and coronary artery calcification in Korean adults. Cardiovasc Diabetol. 2017;16(1):108.

24. Wang $Y$, Wang $Y$, Zhao $X$, et al. Clopidogrel with aspirin in acute minor stroke or transient ischemic attack. N Engl J Med. 2013;369(1):11-9.

25. Samuels OB, Joseph GJ, Lynn MJ, Smith HA, Chimowitz MI. A standardized method for measuring intracranial arterial stenosis. AJNR Am J Neuroradiol. 2000;21(4):643-6.

26. Chen W, Pan Y, Jing J, et al. Recurrent Stroke in Minor Ischemic Stroke or Transient Ischemic Attack With Metabolic Syndrome and/or Diabetes Mellitus. J Am Heart Assoc. 2017;6(6):e005446.

27. Lo JW, Crawford JD, Samaras K, et al. Association of Prediabetes and Type 2 diabetes with cognitive function after stroke: a STROKOG collaboration study. Stroke. 2020;51(6):1640-6.

28. Rao Z, Zheng H, Wang F, et al. The association between high on-treatment platelet reactivity and early recurrence of ischemic events after minor stroke or TIA. Neurol Res. 2017;39(8):719-26.

29. Yang Y, Chen W, Pan Y, et al. Effect of ticagrelor versus clopidogrel on platelet reactivity measured by thrombelastography in patients with minor stroke or TIA. Aging (Albany NY). 2020;12(20):20085-94.

30. Du Z, Xing L, Lin M, Sun Y. Estimate of prevalent ischemic stroke from triglyceride glucose-body mass index in the general population. BMC Cardiovasc Disord. 2020;20(1):483.

31. Ferrannini E, Barrett EJ, Bevilacqua S, DeFronzo RA. Effect of fatty acids on glucose production and utilization in man. J Clin Invest. 1983:72(5):1737-47.

32. Kain K, Catto AJ, Grant PJ. Associations between insulin resistance and thrombotic risk factors in high-risk south Asian subjects. Diabet Med. 2003:20(8):651-5. 
33. Deng $X L$, Liu Z, Wang C, Li Y, Cai Z. Insulin resistance in ischemic stroke. Metab Brain Dis. 2017;32(5):1323-34.

34. Lu H, Lei S, Zhao J, Chen N. Platelet activity in Chinese obese adolescents with and without insulin resistance. J Thromb Thrombolysis. 2014;37(2):165-70.

35. Zhang $X$, Zhu Y, Song F, et al. Effects of purified anthocyanin supplementation on platelet chemokines in hypocholesterolemic individuals: a randomized controlled trial. Nutr Metab (Lond). 2016;13:86.

36. Li Y, Li X, Zhang Y, et al. Impact of glycemic control status on patients with ST-segment elevation myocardial infarction undergoing percutaneous coronary intervention. BMC Cardiovasc Disord. 2020;20(1):36.

37. Antithrombotic TC. Collaborative meta-analysis of randomised trials of antiplatelet therapy for prevention of death, myocardial infarction, and stroke in high risk patients. BMJ. 2002;324(7329):71-86

38. James S, Angiolillo DJ, Cornel JH, et al. Ticagrelor vs. clopidogrel in patients with acute coronary syndromes and diabetes: a substudy from the PLATelet inhibition and patient outcomes (PLATO) trial. Eur Heart J. 2010;31(24):3006-16.

39. Patrono C, Baigent C, Hirsh J, Roth G. Antiplatelet drugs: American College of Chest Physicians Evidence-Based Clinical Practice Guidelines (8th edition). Chest. 2008;133(6 Suppl):199S-233S.
40. Moore SF, Williams CM, Brown E, et al. Loss of the insulin receptor in murine megakaryocytes/platelets causes thrombocytosis and alterations in IGF signalling. Cardiovasc Res. 2015;107(1):9-19.

41. Topcuoglu MA, Arsava EM, Ay H. Antiplatelet resistance in stroke. Expert Rev Neurother. 2011;11(2):251-63.

42. Paven E, Dillinger JG, Bal Dit Sollier C, et al. Determinants of aspirin resistance in patients with type 2 diabetes. Diabetes Metab. 2020;46(5):370-6.

43. Jia W, Jia Q, Zhang Y, Zhao X, Wang Y. Association between insulin resistance and aspirin or clopidogrel resistance in Chinese patients with recent ischemic stroke/TIA. Neurol Res. 2021;43(5):406-11.

44. Lundström A, Laska A-C, Von Arbin M, Jörneskog G, Wallén H. Glucose intolerance and insulin resistance as predictors of low platelet response to clopidogrel in patients with minor ischemic stroke or TIA. Platelets. 2013;25(2):102-10

\section{Publisher's Note}

Springer Nature remains neutral with regard to jurisdictional claims in published maps and institutional affiliations.
Ready to submit your research? Choose BMC and benefit from:

- fast, convenient online submission

- thorough peer review by experienced researchers in your field

- rapid publication on acceptance

- support for research data, including large and complex data types

- gold Open Access which fosters wider collaboration and increased citations

- maximum visibility for your research: over $100 \mathrm{M}$ website views per year

At BMC, research is always in progress.

Learn more biomedcentral.com/submissions 